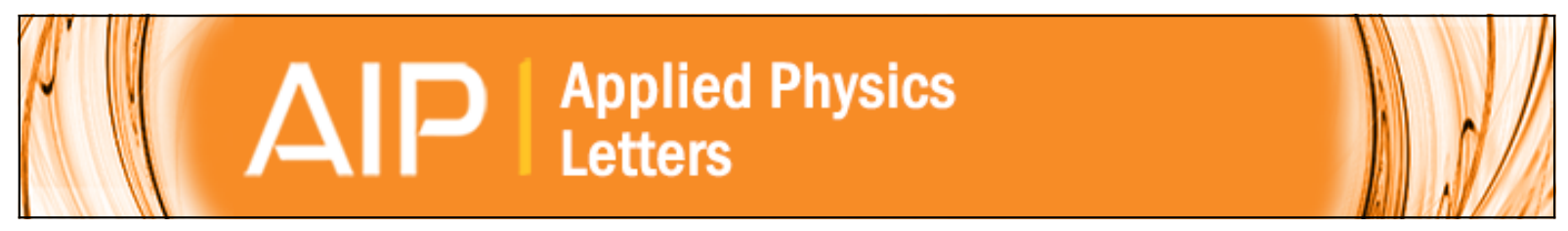

\title{
Hydrogen desorption in SiGe films: A diffusion limited process
}

J. Vizoso, F. Martín, J. Suñé, and M. Nafría

Citation: Applied Physics Letters 70, 3287 (1997); doi: 10.1063/1.118429

View online: http://dx.doi.org/10.1063/1.118429

View Table of Contents: http://scitation.aip.org/content/aip/journal/apl/70/24?ver=pdfcov

Published by the AIP Publishing

\section{Instruments for advanced science}
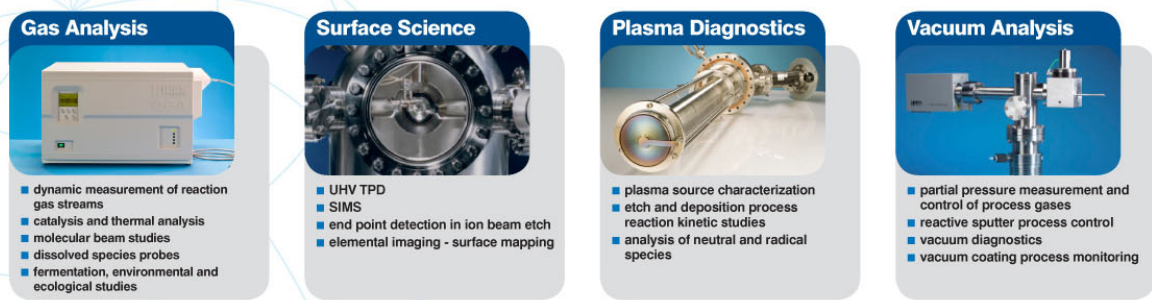

contact Hiden Analytical for further details

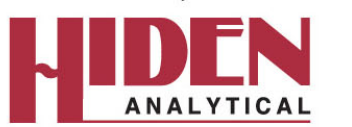

info@hideninc.com www.HidenAnalytical.com CLICK to view our product catalogue 


\title{
Hydrogen desorption in SiGe films: A diffusion limited process
}

\author{
J. Vizoso, ${ }^{\text {a) }}$ F. Martín, J. Suñé, and M. Nafría \\ Departament d'Enginyeria Electrònica, Universitat Autònoma de Barcelona, \\ 08193-Bellaterra, Barcelona, Spain
}

(Received 2 August 1996; accepted for publication 14 April 1997)

\begin{abstract}
A model to explain the hydrogen desorption kinetics in SiGe alloys is presented. This is an extension of a previous desorption model of hydrogen from $\mathrm{Si}$, that considers the presence of three dimer types in the surface in which hydrogen atoms tend to pair before the desorption reaction. Surface diffusion is included in the model. The comparison with experimental results shows that desorption is a diffusion limited process. (C) 1997 American Institute of Physics.
\end{abstract}

[S0003-6951(97)02724-1]

From the recent progress of different techniques for the growth of very thin semiconductor films, much effort has been dedicated to the epitaxy of $\mathrm{Si}_{1-x} \mathrm{Ge}_{x}$ alloys on silicon due to the possibility of tailoring the band gap. This has opened the door to the fabrication of new heterostructure based devices within the framework of the existing Si process technology, with applications in the field of high speed electronics and optoelectronics. ${ }^{1-3}$ Among these techniques, those based on temperature limited reaction process (LRP) or rapid thermal chemical vapor deposition (RTCVD) have been found to provide very thin heteroepitaxial layers with sharp transition regions. ${ }^{4,5}$ However, in order to have good control over layer thickness, a knowledge of the growth kinetics is necessary. In this regard, several works have dealt with the effects of the growth conditions and composition on the kinetics of the $\mathrm{Si}_{1-x} \mathrm{Ge}_{x}$ epitaxy, ${ }^{5-9}$ concluding that at low temperatures the growth rate of epitaxial layers is controlled by the presence of hydrogen atoms in the surface, which results from the decomposition of the molecules of the precursor gases (usually $\mathrm{SiH}_{4}$ and $\mathrm{GeH}_{4}$ ) during the deposition process, and whose desorption is not immediate. In addition, the growth rate at low temperatures is considerably higher than in pure $\mathrm{Si}$, which is attributed to a catalytic effect on the desorption of hydrogen produced by the presence of Ge. ${ }^{10}$ From these comments, it follows that the understanding of the epitaxial growth of $\mathrm{Si}_{1-x} \mathrm{Ge}_{x}$ films begins by the study of the desorption kinetics of hydrogen from the semiconductor surface.

In this letter a model for hydrogen desorption in $\mathrm{Si}_{1-x} \mathrm{Ge}_{x}$ (100) is proposed. It is based on the following assumptions: (1) in the surface, atoms pair up forming three types of dimers: $\mathrm{Si}-\mathrm{Si}, \mathrm{Ge}-\mathrm{Ge}$, and $\mathrm{Si}-\mathrm{Ge}$. This is supported by the fact that in pure $\mathrm{Si}$ and $\mathrm{Ge}$, dimerization in the surface occurs. ${ }^{11-14}$ The dimers can be found in three possible states of hydrogen occupancy: clean ("unoccupied dimers"), characterized by a $\pi$ interaction between the atoms forming the dimer, singly occupied (where hydrogen adsorption breaks the double bond resulting in a $\mathrm{Si}-\mathrm{H}$ or $\mathrm{Ge}-\mathrm{H}$ bond and an unpaired dangling bond), and doubleoccupied. (2) Hydrogen desorption occurs by pre-pairing of two hydrogen atoms in a single dimer. This has been suggested by several authors ${ }^{15,16}$ to explain the first-order kinetics of hydrogen desorption in pure $\mathrm{Si}$, and has been sup-

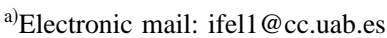

ported by Boland $^{12}$ by arguing that there exists a thermodynamic preference for the hydrogen atoms to pair as a direct consequence of the existence of a $\pi$ bond between the atoms in unoccupied dimers. (3) The distribution of double occupied dimers depends on two processes: desorption and diffusion in the surface. It is assumed that desorption occurs at a different rate from each type of dimer, so that three desorption constants $\left(K_{\mathrm{Si}}, K_{\mathrm{Ge}}\right.$, and $\left.K_{\mathrm{SiGe}}\right)$ are considered. The temperature dependence of these constants is of the Arrhenius type $\left[K_{i}=\nu_{i} \exp \left(-E_{a i} / K T\right)\right], \nu_{i}$ being a frequency factor and $E_{a i}$ the thermal activation energy associated to each process, with is given by $E_{\mathrm{X} 1-\mathrm{H}}+E_{\mathrm{X} 2-\mathrm{H}}-E_{\pi}$ $-E_{\mathrm{H}-\mathrm{H}} ; E_{\pi}$ is the $\pi$ bond energy, and the other terms the energies corresponding to the indicated bonds, where $\mathrm{X} 1$ and $\mathrm{X} 2$ refer to silicon or germanium atoms, depending on the dimer from which desorption occurs. Desorption tends to destroy the thermodynamic equilibrium distribution of dimers, which is reestablished by means of diffusion on the surface. If diffusion is considered to be a very fast process as compared to desorption, the occupancy state of the dimers is given by a thermodynamic equilibrium distribution which depends only on temperature and hydrogen coverage, $\theta$. This fast diffusion assumption is considered by D'Evelyn, Yang, and $\mathrm{Sutcu}^{16}$ in modeling the desorption of hydrogen from pure $\mathrm{Si}$. However, in order to be valid even at low temperature, diffusion will be included in our model. Besides, since the hydrogen desorption kinetics are faster for $\mathrm{SiGe}$ films, ${ }^{8,10,17}$ the prior assumption of instantaneous diffusion is doubtful.

According to the previous assumptions, we postulate that the desorption rate of hydrogen is given by

$$
\frac{d \theta}{d t}=-K_{\mathrm{Si}} \theta_{2}^{\mathrm{Si}}-K_{\mathrm{Ge}} \theta_{2}^{\mathrm{Ge}}-K_{\mathrm{SiGe}} \theta_{2}^{\mathrm{SiGe}},
$$

where $\theta_{2}^{\mathrm{Si}}, \theta_{2}^{\mathrm{Ge}}$, and $\theta_{2}^{\mathrm{SiGe}}$ are the hydrogen coverage in doubly occupied dimers of $\mathrm{Si}, \mathrm{Ge}$, and $\mathrm{SiGe}$, respectively. On the other hand, the evolution of doubly occupied dimers for each species is governed by

$$
\begin{aligned}
& \frac{d \theta_{2}^{\mathrm{Si}}}{d t}=-K_{\mathrm{dif}}\left(\theta_{2}^{\mathrm{Si}}-\theta_{2 \mathrm{est}}^{\mathrm{Si}}\right)-K_{\mathrm{Si}} \theta_{2}^{\mathrm{Si}}, \\
& \frac{d \theta_{2}^{\mathrm{Ge}}}{d t}=-K_{\mathrm{dif}}\left(\theta_{2}^{\mathrm{Ge}}-\theta_{2 \mathrm{est}}^{\mathrm{Ge}}\right)-K_{\mathrm{Ge}} \theta_{2}^{\mathrm{Ge}},
\end{aligned}
$$




$$
\frac{d \theta_{2}^{\mathrm{SiGe}}}{d t}=-K_{\mathrm{dif}}\left(\theta_{2}^{\mathrm{SiGe}}-\theta_{2 \mathrm{est}}^{\mathrm{SiGe}}\right)-K_{\mathrm{SiGe}} \theta_{2}^{\mathrm{SiGe}}
$$

where the first term on the right-hand side is related to diffusion and tends to reestablish the thermodynamic equilibrium distribution at a rate given by $K_{\text {dif }}$, whereas the second term corresponds to desorption. $\theta_{\text {2est }}^{i}$ is the equilibrium distribution of doubly occupied dimers and can be inferred from an extension of the lattice gas model of D'Evelyn, Yang, and Sutcu. ${ }^{16}$ Let $N=N_{\mathrm{Si}}+N_{\mathrm{Ge}}+N_{\mathrm{SiGe}}$ be the total number of dimers in the surface, where each individual contribution is assumed to be statistically determined from the percentage of $\mathrm{Ge}$ in the alloy. For Si and Ge dimers, there are three possible states of occupancy. However, for SiGe dimers, four states arise, provided that there are two distinguishable singly occupied states. If $n_{i}^{j}$ is the number of dimers of type $j$ and occupancy $i$, and $E_{i}^{j}$ the corresponding energy, the partition function $Z$ is given by

$$
\begin{aligned}
Z= & \frac{N_{\mathrm{Si}}^{!}}{n_{0}^{\mathrm{Si}} ! n_{1}^{\mathrm{Si}} ! n_{2}^{\mathrm{Si}} !} \frac{N_{\mathrm{Ge}}^{!}}{n_{0}^{\mathrm{Ge}} ! n_{1}^{\mathrm{Ge}} ! n_{2}^{\mathrm{Ge}} !} \\
& \times \frac{N_{\mathrm{SiGe}}^{!}}{n_{0}^{\mathrm{SiGe}} ! n \frac{\mathrm{SiGe}}{1} ! n_{1}^{\mathrm{SiGe}} ! n_{2}^{\mathrm{SiGe}} !} 2^{\left(n_{1}^{\mathrm{Si}}+n_{1}^{\mathrm{Ge}}\right)} \\
& \times \exp \left(-\beta \sum_{i=1,2 ; j} n_{i}^{j} E_{i}^{j}\right)
\end{aligned}
$$

where it has been assumed that the energy reference level is given by the clean dimers, and $\beta=1 / K T$. In the notation of Eq. (5), the underline in singly occupied SiGe dimers indicate the atom at which hydrogen is bounded. If we suppose that $2 M$ hydrogen atoms are chemisorbed on the surface, the following constraints apply to Eq. (5):

$$
\begin{aligned}
& 2 M=2\left(n_{2}^{\mathrm{Si}}+n_{2}^{\mathrm{Ge}}+n_{2}^{\mathrm{SiGe}}\right)+n_{1}^{\mathrm{Si}}+n_{1}^{\mathrm{Ge}}+n \frac{\mathrm{SiGe}}{1}+n_{1}^{\mathrm{SiGe}}, \\
& N_{\mathrm{Si}}=n_{0}^{\mathrm{Si}}+n_{1}^{\mathrm{Si}}+n_{2}^{\mathrm{Si}}, \\
& N_{\mathrm{Ge}}=n_{0}^{\mathrm{Ge}}+n_{1}^{\mathrm{Ge}}+n_{2}^{\mathrm{Ge}}, \\
& N_{\mathrm{SiGe}}=n_{0}^{\mathrm{SiGe}}+n n_{1}^{\mathrm{SiGe}}+n_{1}^{\mathrm{SiGe}}+n_{2}^{\mathrm{SiGe}} .
\end{aligned}
$$

By maximizing $Z$, subject to these constraints, the thermodynamic equilibrium distribution of doubly occupied dimers as a function of hydrogen coverage and temperature can be numerically evaluated. In the model, the energies associated with each dimer state are given by $E_{\pi}-E_{\mathrm{X}-\mathrm{H}}$ and $E_{\pi}$ $-2 E_{\mathrm{X}-\mathrm{H}}$ for singly and doubly occupied dimers of $\mathrm{Si}$ and $\mathrm{Ge}$ (X represents a $\mathrm{Si}$ or Ge atom), and by $E_{\pi}-E_{\mathrm{X}-\mathrm{H}}$ and $E_{\pi}$ $-E_{\mathrm{Si}-\mathrm{H}}-E_{\mathrm{Ge}-\mathrm{H}}$ for the singly and double occupied SiGe dimers. $E_{\pi}$, which is assumed to be independent of the dimer type, ${ }^{11}$ has been obtained from the work of D'Evelyn, Yang, and Sutcu, ${ }^{16}$ and the bond energies $\left(E_{\mathrm{Si}-\mathrm{H}}\right.$ and $\left.E_{\mathrm{Ge}-\mathrm{H}}\right)$ from the values of the activation energies for desorption in pure $\mathrm{Si}^{18}$ and Ge. ${ }^{11}$ In such a way, the energies of the dimer states have been evaluated.

The parameters of the presented model are the hydrogen diffusion constant and the frequency factors. Even though the frequency factor must be dependent on the dimer type, the close proximity of reported values for pure $\mathrm{Si}$ and $\mathrm{Ge}$ leads us to consider a single frequency factor. In such a way,

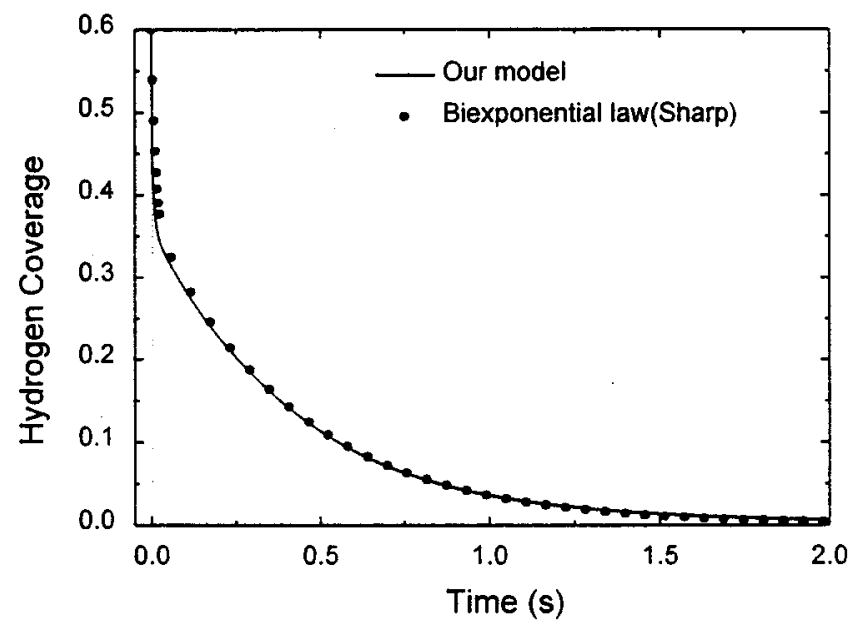

FIG. 1. Desorption kinetics for hydrogen in SiGe. The dots correspond to the biexponential law proposed by Sharp and Eres (Ref. 13) to fit their experimental results. These data have been used to get the parameters of the model proposed in the work.

the parameters of the model are reduced to two. We have fitted this parameter to describe the desorption experiments performed by Sharp and Eres. ${ }^{13}$ A random distribution of dimer states has been chosen to startup the simulation of the desorption process, being the initial coverage $\theta=0.6$. They found that hydrogen coverage exhibits a binary decay with a fast component in the initial stages which prevails only during $\sim 30 \mathrm{~ms}$. This behavior suggests that diffusion is not able to reestablish thermodynamic equilibrium instantaneously. If this was the case, two different stages would not be observed, as has been verified by computer simulation of the desorption process under a wide range of diffusion constants. The best fit (Fig. 1) provides $K_{\text {dif }}=6 \mathrm{~s}^{-1}$ and $\nu=8$ $\times 10^{14} \mathrm{~s}^{-1}$, from which the desorption constants from $\mathrm{Si}$, $\mathrm{SiGe}$, and Ge dimers are 3, 147, and $7187 \mathrm{~s}^{-1}$, respectively. This confirms our assumption that hydrogen desorption is a diffusion limited process because the diffusion rate is much smaller than the desorption rates from SiGe and Ge dimers.

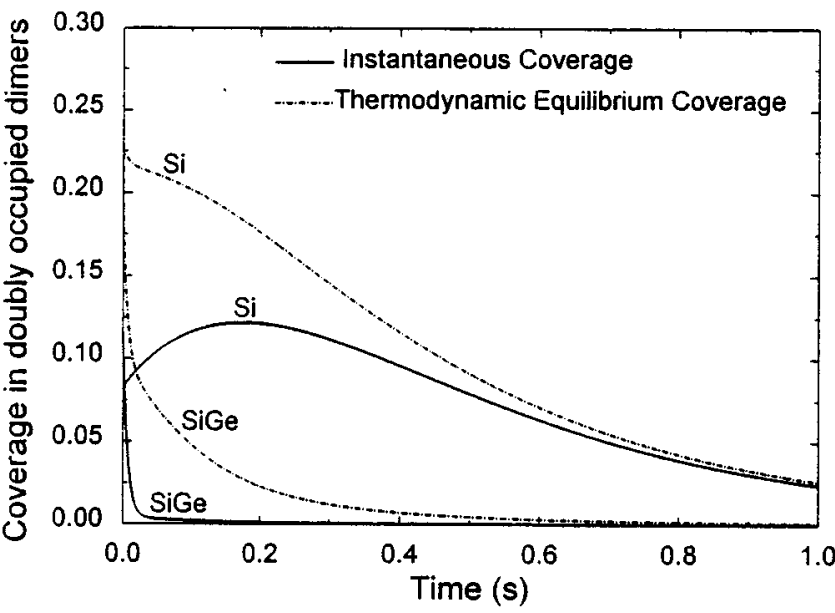

FIG. 2. Evolution of hydrogen coverage in double occupied dimers. For comparison, the thermodynamic equilibrium distribution is also represented. The decay of double occupied Ge dimers is not visible in the time scale. 
On the other hand, the fact that desorption is a two-step process is related, according to our model, to the high $K_{\mathrm{Ge}}$ constant, which is responsible for a very fast decay of doubly occupied Ge dimers that cannot be appreciated in the time scale of Fig. 1, resulting in a desorption in two stages, instead of three. Figure 2 shows the time evolution of doubly occupied dimers. It is interesting to note that the duration of the first step of Fig. 1 perfectly correlates with the decay time of doubly occupied SiGe dimers. Therefore, it can be concluded that the fast first step of Fig. 1 is due to the complete desorption of hydrogen from SiGe and Ge dimers, which is very fast since the desorption constants are very high compared to the rate at which diffusion is able to supply states of double occupancy for these dimer sites. The binary decay is then related to a diffusion controlled process and is a consequence of the desorption of hydrogen in a sequential fashion, where in a first step hydrogen desorption occurs from double occupied SiGe and Ge dimers only, and then hydrogen decay is governed by $\mathrm{Si}$ dimers.

In conclusion, we have presented a model for hydrogen desorption in SiGe alloys based on the pre-pairing of hydrogen atoms in dimer units previous to the desorption reaction. The fast diffusion assumption of hydrogen atoms in the surface of previous works has been suppressed. The model explains the two-step desorption kinetics observed by Sharp and Eres as a result of a diffusion limited desorption of hydrogen from the surface.
The authors thank Dr. J. Camacho for helpful discussions.

${ }^{1}$ M. Arienzo, J. H. Comfort, E. F. Grabbé, D. L. Harame, S. S. Iyer, V. P. Kesan, B. S. Meyerson, G. L. Patton, J. M. C. Stork, and Y.-C. Sun, Microelectron. Eng. 19, 519 (1992).

${ }^{2}$ S. S. Iyer, G. L. Patton, J. M. C. Stork, B. S. Meyerson, and D. L. Harame, IEEE Trans. Electron Devices ED-36, 2043 (1989).

${ }^{3}$ H. Morkoç, B. Sverdlov, and G. Gao, Proc. IEEE 81, 493 (1993).

${ }^{4}$ C. M. Gronet, C. A. King, W. Opyd, J. F. Gibbons, S. D. Wilson, and R. Hull, J. Appl. Phys. 61, 2407 (1987).

${ }^{5}$ D. Dutartre, P. Warren, I. Berbezier, and P. Pettet, Thin Solid Films 222, 52 (1992).

${ }^{6}$ Y. Zhong, M. C. Öztúrk, D. T. Grider, J. J. Wortmann, and M. A. Littlejohn, Appl. Phys. Lett. 57, 2092 (1990).

${ }^{7}$ S. M. Jang and R. Reif, Appl. Phys. Lett. 60, 707 (1992).

${ }^{8}$ S. Gu, Y. Zheng, R. Zhang, R. Wang, and P. Zhong, J. Appl. Phys. 75, 5382 (1994)

${ }^{9}$ M. K. Sanganeria, K. E. Violette, M. C. Öztürk, G. Harris, and D. M. Maher, J. Electrochem. Soc. 142, 285 (1995).

${ }^{10}$ B. M. Ning and J. E. Crowell, Appl. Phys. Lett. 60, 2914 (1992).

${ }^{11}$ S. M. Cohen, Y. L. Yang, E. Rouchouze, T. Jin, and M. P. D'Evelyn, J. Vac. Sci. Technol. A 10, 2166 (1992).

${ }^{12}$ J. J. Boland, J. Vac. Sci. Technol. A 10, 2458 (1992).

${ }^{13}$ J. W. Sharp and G. Eres, Appl. Phys. Lett. 62, 2807 (1993).

${ }^{14}$ A. Oral and R. Ellialtioglu, Surf. Sci. 323, 295 (1995).

${ }^{15}$ M. L. Wise, B. G. Koehler, P. Gupta, P. A. Coon, and S. M. George, Surf. Sci. 258, 166 (1991).

${ }^{16}$ M. P. D'Evelyn, Y. L. Yang, and L. F. Sutcu, J. Chem. Phys. 96, 852 (1992).

${ }^{17}$ D. J. Robbins, J. L. Glasper, A. G. Cullis, and W. Y. Leong, J. Appl. Phys. 69, 3729 (1991).

${ }^{18}$ K. Sinniah, M. G. Sherman, L. B. Lewis, W. H. Weinberg, J. T. Yates, and K. C. Janda, Phys. Rev. Lett. 62, 567 (1989). 